\title{
Nivel de competencias pedagógicas docentes percibidas por estudiantes universitarios
}

\section{Level of teaching pedagogical competences perceived by university students}

\author{
Mely Ruiz-Aquinol,a* \\ https://orcid.org/0000-0002-8340-7898 \\ Tania Chavez Calderón ${ }^{2}$ \\ https://orcid.org/0000-0002-0983-6029, \\ Yaneth Correa Sánchez² \\ https://orcid.org/0000-0002-9914-0358
}

\author{
Deyson Mayta Arias², \\ https://orcid.org/0000-0001-5231-8553 \\ Gabriela Minaya Revolledo ${ }^{2}$ \\ https://orcid.org/0000-0003-0120-0170 \\ Eler Borneo Cantaliciol,a \\ https://orcid.org/0000-0002-6273-9818
}

\section{Citar como}

Ruiz-Aquino, M., Chavez T., Correa, Y., Mayta, D., Minaya, G. y Borneo, E. (2020). Nivel de competencias pedagógicas docentes percibidas por estudiantes universitarios. Desafíos, 2020; 11(1); 11-8. https://doi.org/10.37711/desafios.2020.11.1.134

\section{RESUMEN}

Objetivo. Identificar el nivel de competencias pedagógicas de los docentes universitarios percibidos por estudiantes de la Universidad de Huánuco (UDH), 2020. Métodos. Fue un estudio transversal, observacional y descriptivo. La población estuvo compuesta por 7853 estudiantes, siendo la muestra unos 350 estudiantes seleccionados por un muestreo probabilístico, aleatorio y por estratos. Los instrumentos de medición fueron la guía de encuesta de características demográficas y académicas de la muestra y la escala de las competencias pedagógicas de los docentes, según la percepción del estudiante. Este último instrumento fue previamente validado a nivel cualitativo y cuantitativo. Para el análisis inferencial se empleó la prueba chi cuadrado para una muestra. Resultados. Según los estudiantes universitarios, los niveles de competencias pedagógicas de los docentes universitarios se percibieron mayoritariamente (72,9\%) en un nivel bajo de competencias; de acuerdo a las dimensiones, el 72,6 \% sostuvieron competencias básicas bajas, el $82,6 \%$ manifestaron competencias específicas bajas y el 85,4\% indicaron competencias transversales bajas. Por otro lado, existieron diferencias estadísticamente significativas, dependiendo del nivel de competencias pedagógicas (altas, moderadas, bajas), con $p \leq 0,000$. Conclusión. El nivel de competencias pedagógicas de los docentes universitarios según la percepción de los estudiantes en mayor proporción es percibido en un nivel bajo.

Palabras clave: competencias del docente, profesor de enseñanza superior, estudiante universitario, calidad de la educación, enseñanza, aprendizaje.

\footnotetext{
ABSTRACT

Objective. To Identify the level of pedagogical competencies of university teachers perceived by students of Universidad de Huánuco (UDH), 2020. Methods. It was a cross-sectional, observational and descriptive study. Population comprised 7853 students, with a sample of 350 students selected by probabilistic, random and strata sampling. The measurement instruments were a survey guide of demographic and academic characteristics of the sample, and a scale of the pedagogical competences of teachers, according to student's perception. This last instrument was previously validated at a qualitative and quantitative level. For the inferential analysis, chi-square test was used for one sample. Results. According to university students, the level of pedagogical competences of university teachers were perceived in the majority (72.9\%) at a low level. According to dimensions, $72.6 \%$ had low basic competences, $82.6 \%$ showed low specific competences and $85.4 \%$ indicated low transversal competences. On the other hand, there were statistically significant differences, depending on the level of pedagogical competences (high, moderate, low), with $p \leq 0.000$. Conclusion. The level of pedagogical competences of university teachers according to the perception of students in a greater proportion is perceived at a low level.

Keywords: pedagogical competences, university teachers, university students, education quality, teaching, learning.
}

\footnotetext{
Filiación y grado académico

1 Universidad de Huánuco, Huánuco, Perú.

2 Estudiante de Enfermería en la Universidad de Huánuco, Perú.

a Magister en Salud Pública y Gestión Sanitaria.
} 


\section{INTRODUCCIÓN}

Durantelosúltimosaños, los países latinoamericanos se han convertido en vitrina de transformación para la educación, debido a los grandes avances en la aplicación de las políticas educativas, involucrando a todos los actores educativos (Gálvez y Milla 2018). Al respecto, Moscoso y Hernández (2015) explican que la enseñanza universitaria viene afrontando demandas y desafíos que exigen revisar la formación del profesor universitario; el cual debe tener un profundo dominio de la especialidad que imparte, pero también una sólida formación pedagógica que le posibilite realizar de una forma más eficiente su labor educativa.

La educación superior universitaria peruana viene experimentando mejoras significativas a través de sucesivas reformas, con respecto a décadas anteriores, pues disponen de mucha más información, tienen más criticidad, no solo acerca del contenido que reciben en las aulas sino también acerca de la forma como sus docentes les proponen aprender. En estas circunstancias, el estudiante se convierte en evaluador de las competencias de su docente, por lo que es necesario la transformación de las estrategias didácticas de los docentes hacia el enfoque socioformativo para promover que sus estudiantes construyan aprendizajes significativos.

González-Garcia (2014) refiere, además, que muchos docentes experimentan en sus aulas, sin lograr que sus estudiantes construyan aprendizajes significativos. Aunado a la escasa o ninguna formación pedagógica que tienen, muchos son profesionales que poseen una preparación profesional en sus respectivas disciplinas; sin embargo, los que se integran a la docencia y la investigación universitaria llegan a ella sin conocimientos suficientes de los aspectos relativos a la didáctica para la enseñanza de sus materias. Lo cual les dificulta la planificación de su proceso enseñanza aprendizaje; por lo que únicamente se basan en un conocimiento intuitivo y empírico que han heredado de sus antiguos docentes (Moscoso y Hernández, 2015).

Al respecto, Freire (1970), citado por Ruíz y Ortiz (2019), indica que la falta de formación pedagógica del docente genera inseguridad y desconocimiento en el estudiante, limitando la creatividad didáctica y promoviendo, sin desearlo, una "educación bancaria" y, por tanto, la repetición de contenidos con poca o ninguna reflexión o aplicación práctica que estimule el pensamiento crítico. A ello se suma que muchos estudiantes no tienen interés en lo que aprenden, estando más concentrados en "ganar el curso" que en aprender. De este modo, están acostumbrados a la repetición de contenidos, con poca o ninguna reflexión o aplicación práctica que estimule el pensamiento crítico frente a dicha problemática. Por consiguiente, se necesita que las/os docentes universitarios formen estudiantes conscientes de su realidad y capaces de generar nuevos conocimientos con el objeto de formar mejores personas. Tal como recomiendan Ruíz y Ortiz (2019), los egresados de las universidades deben evidenciar desempeños óptimos en la solución de problemas del contexto en el que vivan, en condiciones de insertarse en el mundo social, laboral y tecnológico del presente siglo, con sostenibilidad ambiental, contribuyendo a mejorar las condiciones de vida y actuando con compromiso y respeto en proyectos sociales, mediante la aplicación del pensamiento complejo y el trabajo colaborativo.

En la misma orientación, Pazmiño (2018) sostiene que las competencias son procesos complejos que las personas ponen en acción, actuación y creación con la finalidad de resolver problemas y realizar actividades, para aportar en la construcción y transformación de la realidad; para conseguir lo cual, se necesitará integrar el "saber ser", el "saber conocer" y el "saber hacer". De igual modo, Arboleda (2011) argumenta que las competencias pedagógicas son conocimientos, capacidades, habilidades, destrezas, actitudes y disposiciones que alguien debe poseer para intervenir en la formación integral de un individuo, en el contexto de la enseñanza. Por lo tanto, los docentes deben poseer las competencias pedagógicas necesarias para asumir su tarea en el complejo entorno de los aprendizajes y las comprensiones que deben construir los estudiantes.

Para Fernández et al., (2016), la competencia pedagógica debe ser evaluada mediante el desempeño del docente en la educación superior; lo cual puede ser realizado por diversos métodos: la autoevaluación del mismo docente, la evaluación por la institución y la evaluación por los estudiantes. También, González (2014) alude a que la docencia, como proceso planificado e intencionado, debe promocionar los aprendizajes significativos y la construcción de conocimientos, el desarrollo integral del ser humano, el desarrollo de un pensamiento crítico, científico y creativo; así como la formación de valores humanísticos, mediante un proceso caracterizado por el trabajo en equipo, la responsabilidad compartida y la solidez teórica. El desempeño docente se entiende como la práctica pedagógica observable, se manifiesta cuando el docente expresa su competencia y tiene que ver con el logro de aprendizajes esperados. Asimismo, cabe resaltar que la pedagogía tiene un carácter transdisciplinario; lo cual le proporciona 
la capacidad para acercarse a la realidad y a la solución de los problemas humanos (González, 2014). Respecto a la temática del estudio, Tapia y Tipula (2017) hallaron que las competencias pedagógicas del docente, según la opinión de los estudiantes, se basan más en componentes cognitivos.

En Ecuador, el estudio de Carrillo y Duran (2018) reveló que las competencias de los docentes son de nivel medio a bajo. Del mismo modo, en Barcelona (España), Abadía (2015) encontró que las cuestiones más valoradas en los docentes fueron las competencias comunicativas, las relaciones interpersonales y la metodología; ya secundariamente dieron menos importancia a las competencias de planificación y gestión de la docencia y el trabajo en equipo.

En Lima (Perú), Labajos (2013) identificó que el $75,7 \%$ de los docentes está en proceso de desarrollo de competencias genéricas y el 64,1\% de competencias pedagógicas; un 20,2 \% percibió que el docente evidencia competencias desarrolladas genéricas yel 31,7 \% de competencias desarrolladas pedagógicas; mientras que el 4 \% percibe que los docentes aún no tienen desarrolladas las competencias genéricas ni pedagógicas. En Huánuco, Portocarrero (2018) desarrolló un estudio experimental donde identificó que, antes de la intervención, los docentes obtuvieron 15,5 de calificación y luego de la intervención obtuvieron 17,0 puntos; evidenciándose el incremento de las puntuaciones en fortalecimiento de competencias docentes. El mismo resultado fue para las dimensiones de estrategias metodológicas, técnicas y pedagógicas. La evaluación del desempeño docente es línea temática de investigación a nivel mundial y nacional; en este sentido fue importante porque, según la reforma de la educación superior universitaria, después de licenciamiento, el Ministerio de Educación (MINEDU) y la Superintendencia Nacional de Educación Superior Universitaria (Sunedu) en la Ley N. 30220 (Congreso de la República, 2014) tienen como parte de sus políticas de evaluación periódica que todas las universidades operen por encima de las condiciones básicas de calidad, dentro del eje I: modelo educativo. Por ello, a partir del 2015, las universidades están obligadas a evaluar a sus docentes; y qué mejor que desde la percepción del estudiante, quien es el beneficiario directo de las enseñanzas del docente. Gálvez y Milla (2018), explican que ante los cambios acelerados de la sociedad actual, es necesario priorizar la visión de las políticas educativas establecidas por el Ministerio de Educación; principalmente aquellas que consideren la necesidad de un cambio profundo de la práctica docente.
El desempeño pedagógico del docente y la revalorización son trascendentales e imprescindibles para estructurar el cambio en cualquier política educativa. Por ello este estudio se concretizó, en aras de proponer las estrategias de mejora para el fortalecimiento de las competencias de los docentes; planteando mejoras en las metodologías de enseñanza aprendizaje, que repercutan en el aprendizaje y el buen rendimiento del estudiante y, por ende, en aquellas prácticas que permitan mejorar la calidad del proceso enseñanza-aprendizaje (Murillo, 2010). Cabe resaltar además que el docente tiene que estar dotado de ciertas cualidades profesionales para ofrecer una educación de calidad, saber una competencia establecida previamente y su competencia real, teniendo en consideración que el estudiante es la razón de ser de la educación.

Por todo lo descrito anteriormente, el objetivo del estudio fue identificar el nivel de las competencias pedagógicas de los docentes universitarios, de acuerdo a las percepciones de los estudiantes de una universidad privada de Huánuco.

\section{MÉTODOS}

\section{Tipo y diseño}

Se realizó un estudio de tipo descriptivo, prospectivo y transversal; con diseño descriptivo y con enfoque cuantitativo. El estudio se llevó a cabo en la Universidad de Huánuco durante el primer trimestre del 2020.

\section{Población y muestra}

La población del estudio fueron 7853 estudiantes matriculados en el ciclo de verano, 2020-0, en los programas de estudio de Enfermería, Odontología, Educación, Obstetricia, Psicología, Ingeniería Ambiental, Arquitectura, Ingeniería Civil, Ingeniería de Sistemas, Administración, Contabilidad y Derecho y Ciencias Políticas de la Universidad de Huánuco . La muestra incluyó a 366 estudiantes universitarios, con una tasa de respuesta global del 95,6 \% (350/366). Dicha muestra fue seleccionada mediante un muestreo probabilístico estratificado, donde cada programa de estudio representó un estrato.

\section{Recolección de datos}

Los participantes fueron incluidos de acuerdo a los siguientes criterios de selección: estudiantes de la Universidad de Huánuco matriculados en el ciclo de verano 2020-0, y quienes aceptaron participar voluntariamente. Asimismo se aplicó una guía de encuesta sociodemográfica que incluyó las carac- 
terísticas demográficas y académicas. También, se aplicó la escala de la percepción de competencias pedagógicas de Villaroel y Bruna (2017), adaptada al contexto y realidad del estudio. Este instrumento constó de 3 dimensiones: competencias básicas (competencias cognitivas, competencias sociales, competencias comunicativas, competencias tecnológicas, competencias personales), competencias específicas (habilidades de planificación y organización del curso, habilidades didácticas, habilidades de evaluación), competencias transversales (manejo del ambiente en la sala de clases, reflexión pedagógica e investigación-acción) con un total de 39 ítems.

En cuanto a la elaboración y validación de los instrumentos de medición a nivel cualitativo y cuantitativo; en primer lugar, se revisó la literatura previa existente sobre nuestra temática; posteriormente, la escala de las competencias pedagógicas fue sometida a un juicio de validación por 3 expertos y 2 jueces, quienes dieron algunas sugerencias en la redacción de los îtems. Asimismo, se aplicaron las encuestas piloto con la finalidad de verificar la comprensión de los reactivos de la escala, así como la tasa de no respuestas. Se calculó la fiabilidad mediante el coeficiente Alfa de Crombach, obteniéndose el valor de 0, 975.

\section{Procedimientos}

Se tramitaron los permisos correspondientes para la aplicación de las encuestas. Los participantes fueron invitados a colaborar voluntariamente en estudio, solicitándoles su consentimiento. Aquellos que participaron respondieron individualmente en la universidad y en aquellos horarios de clases cedidas por las cátedras que colaboraron con el estudio. La recogida de datos se realizó mediante una metodología cuantitativa, por medio de encuestas autoadministradas a través de una aplicación masiva, por aulas y con la presencia de encuestadores independientes y capacitados. A lo largo de la administración de los instrumentos de medición, los investigadores permanecieron presentes. Antes de encuestar a los estudiantes, se les hizo constar que los datos derivados de la investigación se utilizarían con fines exclusivamente académico-científicos bajo la Ley Nacional n² 29733 de protección de los datos personales, una vez completados los instrumentos de evaluación.

\section{Análisis de datos}

En el análisis descriptivo, se calcularon las frecuencias absolutas y relativas de las variables categóricas. En el análisis inferencial (contrastación de hipótesis) se aplicó la prueba no paramétrica de chi cuadrado $\left(X^{2}\right)$, considerándose un $p<0,05$ como significativo, apoyados en el SPSS V15.0.

\section{Aspectos éticos}

Por ser un estudio no experimental no se han transgredido los derechos humanos de las personas, ya que, en las encuestas elaboradas e incluidas en el estudio, la identidad permanecerá en absoluta reserva. Además, se tuvieron en cuenta los principios bioéticos (beneficencia, no maleficencia, justicia y autonomía) y toda la información utilizada para su realización fue tratada con total confidencialidad. Se protegió la privacidad de los participantes en el estudio y se garantizó que los datos obtenidos fueran utilizados exclusivamente con fines investigativos; por lo que no se revelaría el nombre de los participantes en el mismo. Previo al reclutamiento para el estudio, se obtuvo el consentimiento informado por escrito de todos los participantes.

\section{RESULTADOS}

Al analizar las características demográficas de la muestra en estudio, hallamos predominio de edad joven (18 a 29 años); la mitad de la muestra en estudio [50,9 \% (178)] eran del género femenino; respecto a las características académicas: el programa de estudios de procedencia, la gran mayoría de ellos [27,4 \% (96)], pertenecían al programa de estudios de ingeniería civil, el 24,3\% (85) estaban cursando el sexto ciclo de estudios. Al analizar las horas de estudio fuera de la programación habitual de clases, una gran proporción de la muestra [76,3\% (267)] indicó estudiar de 1 a 6 horas y respecto al número de asignaturas que llevaba el estudiante durante el periodo del estudio, mayoritariamente fueron menos de 5 cursos (ver tabla 1).

Al analizar las dimensiones de las competencias pedagógicas de los docentes universitarios según la percepción de los de estudiantes, se evidenció un alto predominio de nivel bajo para las competencias básicas, específicas y transversales, seguido de las competencias específicas percibidas con un nivel moderado, por la muestra en estudio (ver figura 1).

Al analizar de forma global el nivel de las competencias pedagógicas desde la óptica del estudiante, se halló que una gran proporción de la muestra (72,9\%), percibió como de nivel bajo las competencias pedagógicas de los docentes universitarios, seguido de un 26,9\% que tuvo una percepción de nivel moderado.

Al comparar el nivel de percepción de las competencias pedagógicas de los docentes universitarios, según las características demográficas y académicas de la muestra en estudio, se encontró que una importante proporción de estudiantes mujeres (40,6\%) percibió un bajo nivel 
Tabla 1.

Características demográficas y académicas de los estudiantes de la UDH, 2020

\begin{tabular}{|c|c|c|}
\hline \multirow{2}{*}{ Características demográficas y académicas } & \multicolumn{2}{|c|}{$\mathrm{n}=350$} \\
\hline & $\mathrm{fi}$ & $\%$ \\
\hline \multicolumn{3}{|l|}{ Grupo de edades } \\
\hline Joven (18 a 29 años) & 339 & 96,9 \\
\hline Adulto (30 a 59 años) & 11 & 3,1 \\
\hline \multicolumn{3}{|l|}{ Género } \\
\hline Masculino & 172 & 49,1 \\
\hline Femenino & 178 & 50,9 \\
\hline \multicolumn{3}{|l|}{ Programa de estudios } \\
\hline Enfermería & 36 & 10,3 \\
\hline Odontología & 8 & 2,3 \\
\hline Psicología & 16 & 4,6 \\
\hline Administración de Empresas & 44 & 12,6 \\
\hline Contabilidad & 25 & 7,1 \\
\hline Derecho y Ciencias Políticas & 59 & 16,9 \\
\hline Arquitectura & 24 & 6,9 \\
\hline Ingeniería Ambiental & 34 & 9,7 \\
\hline Ingeniería Civil & 96 & 27,4 \\
\hline Ingeniería de Sistemas & 8 & 2,3 \\
\hline \multicolumn{3}{|l|}{ Ciclo de estudios } \\
\hline$\|$ & 7 & 2,0 \\
\hline III & 7 & 2,0 \\
\hline IV & 11 & 3,1 \\
\hline V & 51 & 14,6 \\
\hline $\mathrm{VI}$ & 85 & 24,3 \\
\hline VII & 75 & 21,4 \\
\hline VIII & 69 & 19,7 \\
\hline IX & 33 & 9,4 \\
\hline$x$ & 12 & 3,4 \\
\hline \multicolumn{3}{|l|}{ Horas de estudio } \\
\hline Menor o igual a 6 & 267 & 76,3 \\
\hline Más de 6 & 83 & 23,7 \\
\hline \multicolumn{3}{|l|}{$\begin{array}{l}\text { Número de asignaturas en el semestre } \\
\text { académico }\end{array}$} \\
\hline Menor a 5 & 325 & 92,9 \\
\hline 5 a más & 25 & 7,1 \\
\hline
\end{tabular}

Nota. Guía de encuesta de las características demográficas y

académicas de la muestra en estudio.

de competencias pedagógicas de los docentes. En lo que respecta a la de las competencias según los programas de estudio se halló que, en su mayoría, el programa de Derecho y Ciencias Políticas tuvo una baja percepción del nivel de competencias pedagógicas de sus docentes, seguido del programa de Administración de Empresas, quien también expresó una importante proporción (11,7%)

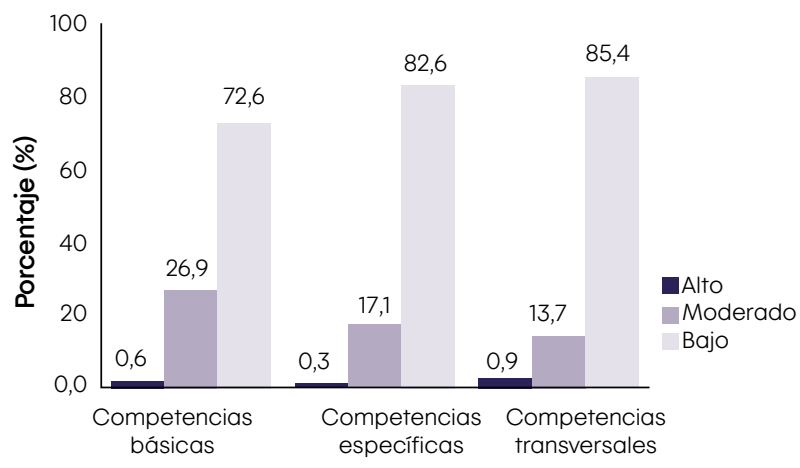

Figura 1. Dimensiones de competencia pedagógicas de los docentes universitarios según la percepción de los estudiantes de la UDH, 2020.

de baja percepción. A diferencia del programa de estudios de Ingeniería Civil que Percibieron, en un $18 \%$, un nivel de competencias de nivel moderado. Respecto a la percepción del nivel de competencias pedagógicas según el ciclo de estudios, un 17,4 \%

Tabla 2.

Nivel de competencias pedagógicas de los docentes universitarios de la UDH, según las características demográficas y académicas de la muestra en estudio, 2020

\begin{tabular}{|c|c|c|c|}
\hline $\begin{array}{c}\text { Características demográficas } \\
\text { y académicas }\end{array}$ & Alto & Moderado & Bajo \\
\hline \multicolumn{4}{|l|}{ Edad en años } \\
\hline Media & 20 & 23 & 23 \\
\hline \multicolumn{4}{|l|}{ Género } \\
\hline Masculino (\%) & 0,0 & 16,9 & 32,3 \\
\hline Femenino (\%) & 0,3 & 10,0 & 40,6 \\
\hline \multicolumn{4}{|l|}{ Programas de estudio } \\
\hline Enfermería (\%) & 0,0 & 1,4 & 8,9 \\
\hline Odontología (\%) & 0,0 & 0,6 & 1,7 \\
\hline Psicología (\%) & 0,0 & 0,0 & 4,6 \\
\hline $\begin{array}{l}\text { Administración de } \\
\text { Empresas (\%) }\end{array}$ & 0,0 & 0,9 & 11,7 \\
\hline Contabilidad (\%) & 0,0 & 1,1 & 6,0 \\
\hline $\begin{array}{l}\text { Derecho y Ciencias } \\
\text { Políticas (\%) }\end{array}$ & 0,0 & 0,3 & 16,6 \\
\hline Arquitectura (\%) & 0,3 & 1,4 & 5,1 \\
\hline Ingeniería Ambiental (\%) & 0,0 & 2,9 & 6,9 \\
\hline Ingeniería Civil (\%) & 0,0 & 18,0 & 9,4 \\
\hline Ingeniería de Sistemas (\%) & 0,0 & 0,3 & 2,0 \\
\hline \multicolumn{4}{|l|}{ Ciclo de estudios } \\
\hline II (\%) & 0,0 & 0,6 & 1,4 \\
\hline III (\%) & 0,0 & 0,9 & 1,1 \\
\hline IV (\%) & 0,0 & 1,4 & 1,7 \\
\hline$\vee(\%)$ & 0,0 & 6,0 & 8,6 \\
\hline VI (\%) & 0,3 & 6,6 & 17,4 \\
\hline VII (\%) & 0,0 & 4,6 & 16,9 \\
\hline VIII (\%) & 0,0 & 2,9 & 16,9 \\
\hline IX (\%) & 0,0 & 3,1 & 6,3 \\
\hline X (\%) & 0,0 & 0,9 & 2,6 \\
\hline
\end{tabular}


Tabla 3.

Comparación del nivel de las competencias pedagógicas de los docentes universitarios según la percepción de los estudiantes de la UDH, 2020

\begin{tabular}{|c|c|c|c|c|}
\hline Nivel de competencia pedagógica & Frecuencia observada & Frecuencia esperada & $\mathbf{X}^{2}$ & $\mathrm{p}$-valor \\
\hline Alto & 1 & 117 & \multirow{4}{*}{283,10} & \multirow{4}{*}{0,000} \\
\hline Moderado & 94 & 117 & & \\
\hline Bajo & 255 & 117 & & \\
\hline Total & 350 & & & \\
\hline
\end{tabular}

Nota. Escala de competencias psicopedagógicas.

del VI percibieron bajas competencias y, a medida que aumenta el ciclo de estudio, se acrecienta la baja percepción.

Por último, al contrastar las hipótesis del estudio se halló que la percepción de las competencias pedagógicas de los estudiantes de la Universidad de Huánuco difieren dependiendo del nivel de competencias pedagógicas de los docentes universitarios, con $\mathrm{p} \leq 0,000$; concluyendo que las competencias pedagógicas de los docentes universitarios, según la percepción de los estudiantes, fueron de nivel bajo (ver tabla 3).

\section{DISCUSIÓN}

Se reconoce que la evaluación del docente universitario es un proceso complejo, que genera diversas manifestaciones y se halla asociado a distintos procedimientos y variables. En este sentido, se planteó el estudio, desde la percepción de los estudiantes universitarios. En este estudio, se halló que los estudiantes tienen una baja percepción de las competencias pedagógicas de los docentes. Este resultado puede ser el producto de haber aplicado y seguir aplicando modelos tradicionales de enseñanza como el enfoque conductista, en la cual los estudiantes cumplen un rol pasivo-receptor del conocimiento; aunque en alguna medida es activo, en el sentido de que aprenden probando, mediante ensayo yerror, "haciendo, experimentando y ensayando". El enfoque conductista parte de que todos y todas las estudiantes aprenden del mismo modo, sin diferencias individuales (Trilla y García, 2001). En tanto, el enfoque de Competencias, a su vez, se encuentra centrado en el hacer y descuida el ser, teniendo baja consideración de los valores y actitudes (Tobón, 2006). Por su parte, en el modelo de evaluación del desempeño docente, centrado en el perfil, se evidencian las características, previamente establecidas por los actores educativos: estudiantes, padres de familia, directivos y colegas; es decir, se enumeran todas aquellas cualidades que debería tener un buen docente, el docente ideal (Gálvez y Milla, 2018).
Asimismo, nuestro estudio se ha basado en el modelo centrado en el comportamiento del docente en el aula que relaciona el comportamiento del docente con el logro de sus estudiantes. Esta relación consiste en crear espacios favorables para el aprendizaje que serán medidos a través de la observación del comportamiento del docente. También nos basamos en el modelo de la práctica reflexiva o de la reflexión-acción fundamentado en la mejora del personal, en una secuencia de etapas para encontrar las debilidades y resolverlas.

Los estudios congruentes a nuestros resultados, como el de Gálvez y Milla (2018), quienes evidenciaron bajos niveles de desempeños de los docentes, relacionados con la preparación del aprendizaje y la discontinuidad de los propósitos en el proceso de evaluación. Al respecto, Falcó (2017) remarca la necesidad de fortalecer la formación inicial de los docentes y promover programas de formación permanente para el desarrollo de esta competencia.

Ramón et al., (2017) hallaron que los docentes tienen claras las conceptualizaciones en torno al enfoque por competencias y sus implicaciones académicas; sin embargo, en la práctica, necesitan mejorar su nivel de dominio en dos competencias: planificar los procesos de enseñanza-aprendizaje atendiendo al enfoque por competencias y evaluar los procesos de enseñanza-aprendizaje con un enfoque formativo. Del mismo modo Álvarez et al., (2018) encontraron que los docentes tutores no estimulan a potenciar las fortalezas del estudiante. Contrario a nuestros resultados Martínez y Guevara (2015) hallaron que el 2,4 \% de la muestra representativa presentó un excelente desempeño docente, el 7,3\% un mal desempeño Docente y el $39 \%$ de los docentes observados se ubican con un buen desempeño docente. En el estudio de Díaz (2017), un 57 \% de estudiantes percibieron que sus docentes tienen buenas competencias. Del mismo modo, Morán (2015) encontró que los docentes poseen un nivel alto de dominio en las competencias relacionadas con la planeación; mientras que para los de didáctica, evaluación y manejo de TIC, los profesores tienen un dominio 
regular. También, Villarroel y Bruna (2017) hallaron que los docentes presentan alto desempeño en los tres niveles de competencia estudiadas: cognitivo, actitudinal y procedimental.

En el mismo contexto Peláez et al., (2017) encontraron que el 57 \% de estudiantes perciben las competencias de los docentes a nivel general como buenas; por lo que una de las ventajas es lograr el licenciamiento de dicha escuela debido a los resultados mostrados. Además, Huacchillo (2019) halló que el 81,6\% considera que la evaluación de las competencias profesionales del docente son eficientes; asimismo se halló que el 48,8 \% (178) considera que la calidad en la formación profesional es muy eficiente. Todo lo cual evidencia que existe relación entre las competencias profesionales del docente y la calidad en la formación profesional, según la percepción de los estudiantes.

Finalmente, concluimos indicando que las competencias pedagógicas de los docentes universitarios, según la percepción de los estudiantes de la Universidad de Huánuco, fueron de nivel bajo, evidenciado en una gran proporción de la muestra. El estudio tiene limitaciones con respecto a la forma de evaluar al docente, siendo que la evaluación es un tema complejo, más aun con los resultados encontrados en un contexto de estudio durante el ciclo verano; sin embargo, se evaluó al docente desde la percepción de los estudiantes mediante una escala de medición de las competencias pedagógicas adaptando a la realidad del estudio y también hubiera sido necesario incluir la variable rendimiento académico o la satisfacción estudiantil, entre otras variables. Frente a esto, sería deseable, por lo tanto, profundizar en la percepción de la evaluación de las competencias pedagógicas de los docentes desde un enfoque de estudio mixto, medido de forma longitudinal al intermedio y al término del semestre académico, estudios comparativos con los resultados de las encuestas que viene aplicando la Universidad de Huánuco a sus estudiantes.

En términos de recomendaciones, es urgente fortalecer las prácticas educativas de los docentes, iniciando ya procesos reflexivos con la participación de los mismos. En suma, la sociedad actual, caracterizada por un alto nivel de complejidad, inestabilidad e incertidumbre, demanda de las universidades una serie de transformaciones, entre las cuales ocupa un lugar prioritario la formación pedagógica del docente universitario, ya que él constituye actor principal del cambio en las universidades en donde formamos profesionales que tienen la misión de impulsar el desarrollo social y científico-tecnológico de la sociedad en su conjunto (Moscoso y Hernández, 2015).
Por último, es necesario el acompañamiento pedagógico, considerando que muchos profesionales que se dedican a la docencia universitaria no tienen la formación pedagógica. Tal como indican Moscoso y Hernández (2015), la formación pedagógica del profesor universitario se corresponde con la necesidad de profesionalizar la docencia universitaria, por lo que es importante entenderla desde una noción de cambio ya que, en la actualidad, hay situaciones cambiantes de contexto social, institucional y de aula que obligan al docente a modificar para mejorar su práctica pedagógica. Ello implica planificar los procesos de enseñanza-aprendizaje, gestionar ambientes agradables de trabajo pedagógico y valorar su profesionalidad. Un acompañamiento pedagógico ayudaría a fortalecer el desempeño del docente, a través de actividades de planificación y previsión. En definitiva, mejorar los aprendizajes de los estudiantes, en busca de la calidad educativa universitaria depende fundamentalmente de la calidad humana, pedagógica y científica de sus docentes.

\section{Agradecimientos}

Esta investigación fue desarrollada como parte de la investigaciónformativa, por ello nuestro agradecimiento al equipo de encuestadores que apoyaron en todo el proceso de la recolección de datos.

\section{REFERENCIAS BIBLIOGRÁFICAS}

Abadía, AR., Bueno, C., Ubieto-Artur, M.I., Márquez, M.D., Sabaté, S., Jorba H. y Pagès, T. (2015). Competencias del buen docente universitario. Opinión de los estudiantes. REDU. Revista de Docencia Universitaria, 13(2), 363-390. Recuperado de https://doi. org/10.4995/redu.2015.5453

Arboleda, J. C. (2011). Hacia un currículo dignificante. En J.C. Arboleda (Ed.). La Pedagogía colombiana. Educación, Pedagogía y Currículo (pp. 9-14). Colombia: REDIPE.

Álvarez-Helle, C., Ortiz-Moreira, L. y Pérez-Villalobos, C. (2018). Percepción de las competencias docentes del tutor clínico según estudiantes de odontología de universidad chilena. Educación Médica Superior, 32(2). Recuperado de http:// scielo.sld.cu/scielo.php?script=sci_arttext\&pi$d=S 0864-21412018000200010 \&$ Ing =es\&tIng=en

Carrillo, J.E., Duran, L.F. (2018). Análisis comparativo de las competencias mediáticas en el desempeño académico de los docentes de las carreras de Pedagogía de la Historia y Ciencias Sociales, Pedagogía de los Idiomas Nacionales y Extranjeros, Pedagogía de las Ciencias Experimentales Mate. J Chem Inf Model, 53(9): 1689-99.

Congreso de la República del Perú. (2014, 3 de julio). Ley n. ${ }^{\circ}$ 30220, Ley Universitaria. Lima, Perú: Congreso de la República del Perú. Diario Oficial El Peruano n. ${ }^{0}$ 12914. http://www.leyes.congreso.gob.pe/Documentos/Leyes/30220.pdf 
Díaz, A. (2006). El enfoque de competencias en la educación: ¿Una alternativa o un disfraz de cambio? Perfiles educativos, 28(111), 7-36. Recuperado de http:// www.scielo.org. $\mathrm{mx} /$ scielo.php? pid = S018526982006000100002\&script=sci_abstract

Fernandes, D.J., Sotolongo, M. y Martínez, C.C. (2016). La Evaluación del Desempeño por Competencias: Percepciones de Docentes y Estudiantes en la Educación Superior. Formación universitaria, 9(5), 15-24. Recuperado de https://dx.doi.org/10.4067/s071850062016000500003

Falcó, J.M. (2017). Evaluación de la competencia digital docente en la Comunidad Autónoma de Aragón. Revista Electrónica de Investigación Educativa, 19(4), 73-83. doi: https://doi.org/10.24320/redie.2017.19.4.1359

González-García, V. (2014) Innovar en docencia universitaria: algunos enfoques pedagógicos. InterSedes, 15(31),51-68. Recuperado de http://www scielo.sa.cr/scielo.php?script=sci_arttext\&pi$d=$ S2215-24582014000200005\&lng=en\&tIng=es.

Gálvez, E. y Milla, R. (2018). Evaluación del desempeño docente: Preparación para el aprendizaje de los estudiantes en el Marco de Buen Desempeño Docente. Propósitos y Representaciones, 6(2), 407-429. Recuperado de https://dx.doi.org/10.20511/pyr2018. v6n2.236

Huacchillo, M.P. (2019). Evaluación de las competencias profesionales del docente y la calidad en la formación profesional según la percepción de los estudiantes de la Facultad de Ciencias de la Salud de la Universidad Privada San Juan Bautista, Chorrillos 2018 (Tesis de maestría). Universidad Nacional de Educación Enrique Guzmán y Valle, Lima, Perú.

Labajos, N.F. Competencias de los docentes de enfermería de la UNMSM según percepción de los estudiantes, 2013 (tesis de pregrado). Recuperado de https://cybertesis.unmsm.edu.pe/handle/20.500.12672/3533

Moscoso, F. y Hernández, A. (2015). La formación pedagógica del docente universitario: un reto del mundo contemporáneo. Revista Cubana de Educación Superior, 34(3), 140-154. Recuperado de http:// scielo.sld.cu/scielo.php?script=sci_arttext\&pi$\mathrm{d}=$ S0257-43142015000300011\&lng=es\&tIng=es

Murillo, H. (2010). Misión del docente: propiciar en el estudiante aprendizajes significativos. Enfermería universitaria, 7(4), 42-52. Recuperado de http://www. scielo.org.mx/scielo.php?script=sci_arttext\&pi$d=\$ 1665-70632010000400007 \& \operatorname{lng}=$ es\&tIng $=e s$

Martínez, G.l. y Guevara, A. (2015). La evaluación del desempeño docente. Ra Ximhai, 11(4), 113-124. https://doi. org/10.35197/rx.11.01.e2.2015.07.gm

Morán, R., Cardoso, E.O., Cerecedo, M.T. y Ortíz, J.C. (2015). Evaluación de las Competencias Docentes de Profesores Formados en Instituciones de Educación Superior: El Caso de la Asignatura de Tecnología en la Enseñanza Secundaria. Formación universitaria, 8(3), 57-64. doi: https://dx.doi.org/10.4067/S071850062015000300007

Peláez, G., Villchez, J., Valderrama, L. y Carbajulca, M. (2017). Evaluación de las competencias del docente bajo la per- cepción de los estudiantes de la escuela profesional de Administración de la Universidad Nacional Santiago Antúnez de Mayolo, 2016. Aporte Santiaguino, 10(1), 119-130. doi: https://doi.org/10.32911/as.2017.v10.n1.188

Pazmiño, J.E. (2018). Capacitación sobre la competencia pedagógica y el conocimiento de estrategias de enseñanza de los docentes de la Universidad Laica VICENTE ROCAFUERTE de Guayaquil-Ecuador, 2016. Yachana,7(1),1-13.Recuperadode http://revistas.ulvr. edu.ec/index.php/yachana/article/view/519/282

Portocarrero-Merino, E. (2018). Cultura pedagógica y competencias del docente universitario desde la percepción del estudiante. Dialnet, 34(1), 135-151.

Ruíz-Aquino, M. y Ortiz, M. (2019). La socioformación un enfoque necesario en la formación superior universitaria. Desafíos, 1(2): 171-80. doi: https://doi. org/10.37711/desafios.2019.1.2.77

Ramón, P., Aquino, S.P. y Alejandro, M. (2017). Evaluación de las competencias docentes de profesores de educación media superior del estado de Tabasco, México. Sinéctica, (48), 2007-7033. Recuperado de http://www.scielo.org.mx/scielo.php?script=sci_arttext\&pid = S1665-109X2017000100013

Tapia, V. y Tipula, F. (2017). Desempeño docente y creencias pedagógicas del profesor universitario en Universidad Toribio Rodríguez de Mendoza de Amazona. Perú.COMUNI@CCIÓN: Revista de Investigación en Comunicación y Desarrollo, 8(2), 72-80.

Trilla, J. y García, E.C. (2001). El legado pedagógico del siglo XX para la escuela del siglo XXI. Barcelona, España: Graó.

Tobón, S. (2006). Aspectos básicos de la formación basada en competencias. Talca: Proyecto Mesesup. Recuperado de https://yavne.edu.mx/pages/circular/ cch/inicio2015/asp_bas_com.pdf

Villarroel, V A, y Bruna, D V. (2017). Competencias Pedagógicas que Caracterizan a un Docente Universitario de Excelencia: Un Estudio de Caso que Incorpora la Perspectiva de Docentes y Estudiantes. Formación universitaria, 10(4), 75-96. https://dx.doi org/10.4067/s0718-50062017000400008

\section{Contribución de los autores}

MRA: diseño y concepción del proyecto, redacción del artículo científico.

TMCC: diseño del proyecto, recolección de datos y redacción del artículo científico.

YRCS: diseño del proyecto, recolección de datos y redacción del artículo científico.

GMR: diseño del proyecto, recolección de datos y redacción del artículo científico.

DDMA: diseño del proyecto, recolección de datos y redacción del artículo científico.

EBC: análisis de datos y elaboración del artículo científico.

Fuentes de financiamiento.

Autofinanciado.

Conflictos de interés

MRA Y EBC, forman parte del equipo editor de la Revista Desafíos, ambos se reservaron de participar en el proceso de revisión.

\section{Correspondencia}

Tania Margot Chavez Calderón

Cel.: 937513359 